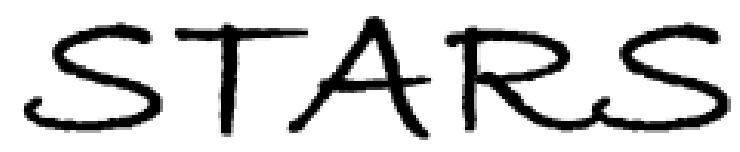

University of Central Florida

STARS

$1-1-2005$

\title{
Infrared laser beam temporal fluctuations: characterization and filtering
}

José M. López-Alonso

Brian Monacelli

University of Central Florida

Javier Alda

Gary D. Boreman

University of Central Florida

Find similar works at: https://stars.library.ucf.edu/facultybib2000

University of Central Florida Libraries http://library.ucf.edu

This Article is brought to you for free and open access by the Faculty Bibliography at STARS. It has been accepted for inclusion in Faculty Bibliography 2000 s by an authorized administrator of STARS. For more information, please contact STARS@ucf.edu.

\section{Recommended Citation}

López-Alonso, José M.; Monacelli, Brian; Alda, Javier; and Boreman, Gary D., "Infrared laser beam temporal fluctuations: characterization and filtering" (2005). Faculty Bibliography 2000s. 5433.

https://stars.library.ucf.edu/facultybib2000/5433

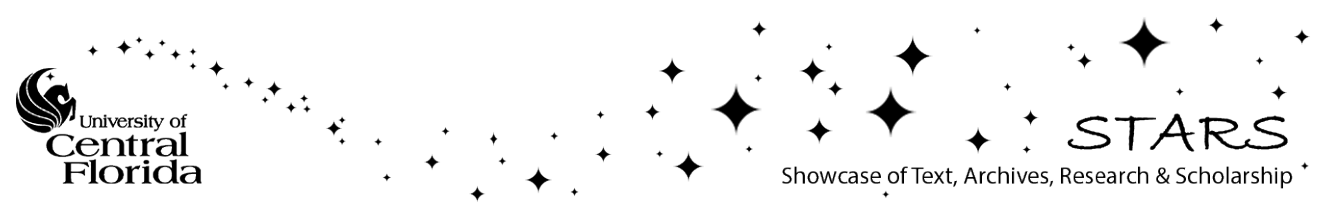




\section{Infrared laser beam temporal fluctuations: characterization and filtering}

José M. López-Alonso

University Complutense of Madrid

School of Optics

Av. Arcos de Jalón

s/n 28037, Madrid, Spain

E-mail: jmlopez@opt.ucm.es

Brian Monacelli, MEMBER SPIE University of Central Florida

College of Optics and Photonics

Orlando, Florida 32816-2700

Javier Alda, MEMBER SPIE

University Complutense of Madrid

School of Optics

Av. Arcos de Jalón

s/n 28037, Madrid, Spain

Glenn D. Boreman, FELLOW SPIE

University of Central Florida

College of Optics and Photonics

Orlando, Florida 32816-2700

\begin{abstract}
IR lasers are widely used in electro-optical applications, especially in detector characterization systems. These lasers can be extremely sensitive to fluctuations in the operational temperature of their cavity and other environmental factors. Due to these influences, the laser output signal normally fluctuates randomly. These variations make it difficult to characterize the laser waist position and exact focus, which in turn causes difficulty with detector measurement. We apply a multivariate statistical approach to characterize and filter these variations and to calculate the "best focus" of a carbon dioxide laser operating at $10.6 \mu \mathrm{m}$. Using this method, the "best focus" can be calculated with great accuracy and can be easily implemented during postsignal processing. Also, this technique can potentially be applied to other situations in which laser signal instability is significant. (๑) 2005 Society of Photo-Optical Instrumentation Engineers. [DOI: 10.1117/1.1905364]
\end{abstract}

Subject terms: infrared lasers; signal processing; principal components.

Paper 040249R received May 6, 2004; revised manuscript received Nov. 22, 2004; accepted for publication Nov. 23, 2004; published online May 23, 2005.

\section{Introduction}

IR lasers are obvious choices for use when characterizing IR detectors. Recently, they have been used in IR antennacoupled detector measurement systems. ${ }^{1-3}$ In these systems, a $\mathrm{CO}_{2}$ laser beam is projected by a suitable optical system onto an IR antenna-coupled bolometer. The output of this detector is studied to characterize its responsivity, noise, and spatial response. Although the $\mathrm{CO}_{2}$ laser is a good source for detector characterization in the IR, its power stability is typically poor. The signal can fluctuate depending on external and internal operational parameters. One of the most critical parameters is the laser cavity temperature. Our laser is stabilized by an external cooler system with its own thermal cycle. Influences of this thermal cycle are evident in the laser beam output.

Coolant fluctuations can also introduce artifacts into the beam shape. These artifacts produce spurious signals and $f$-order moments of laser beams are especially sensitive to this noise. We study the laser output fluctuations to characterize their affects and discern them from the desired data.

Because IR lasers are invisible, alignment of the optical system is a difficult task. The location of the "best focus" is made by searching for the maximum signal along the focal region. The exact beam waist position is not clearly defined, so we take the center of the beam waist to be the "best focus." However, if the laser signal has strong fluctuations, this position is difficult to precisely locate with a single detector.

In this paper, we propose a technique that filters the laser power fluctuations to locate the "best focus." This technique is based on a multivariate statistical approach. This method also provides an estimation of the beam pro-

0091-3286/2005/\$22.00 @ 2005 SPIE file and its second moments through the focal region, with thermal fluctuation noise removed. To acquire the data necessary for this analysis, transverse knife-edge scans are taken through the focal region and postsignal processing is applied.

The paper is organized as follows. The experimental data acquisition setup is described in Sec. 2. Section 3 describes the multivariate statistical technique used to process the data. In Sec. 4, we apply the method to characterize fluctuations of the laser signal. In Sec. 5, a measurement procedure is proposed and applied to a data set with different degrees of resolution.

\section{Experimental Setup}

To characterize small detectors, such as small antennacoupled bolometers, ${ }^{1-4}$ it is desirable to use a test source size that is comparable to the detector size. A carbon dioxide laser is a good choice for antenna-based detector characterization in the long-wave IR (LWIR) because it is spectrally tunable from 9.2 to $11.2 \mu \mathrm{m}$. (For the research of this paper, the laser is operated at $\lambda=10.6 \mu \mathrm{m}$, its most powerful line.) For IR radiation at this wavelength, diffraction limits reasonably attainable source spot sizes to $d_{\text {spot }}$ $=2.44 \lambda(F / \#)$. It has been shown ${ }^{3,4}$ that the antennacoupled detectors act as IR point receivers, with a spatial response of the order of $1 \mu \mathrm{m}, F / 1$ optics are used to obtain an extremely tight focus for the accurate characterization of these detectors.

The system shown in Fig. 1 was designed and characterized to test IR antenna-coupled detectors. The optical system is essentially a Keplerian telescope that is used to expand the IR laser beam to a 50-mm diameter, filling the last two lenses. This collimated output of the telescope is then focused by a 50-mm aspheric lens. Thus, the compos- 


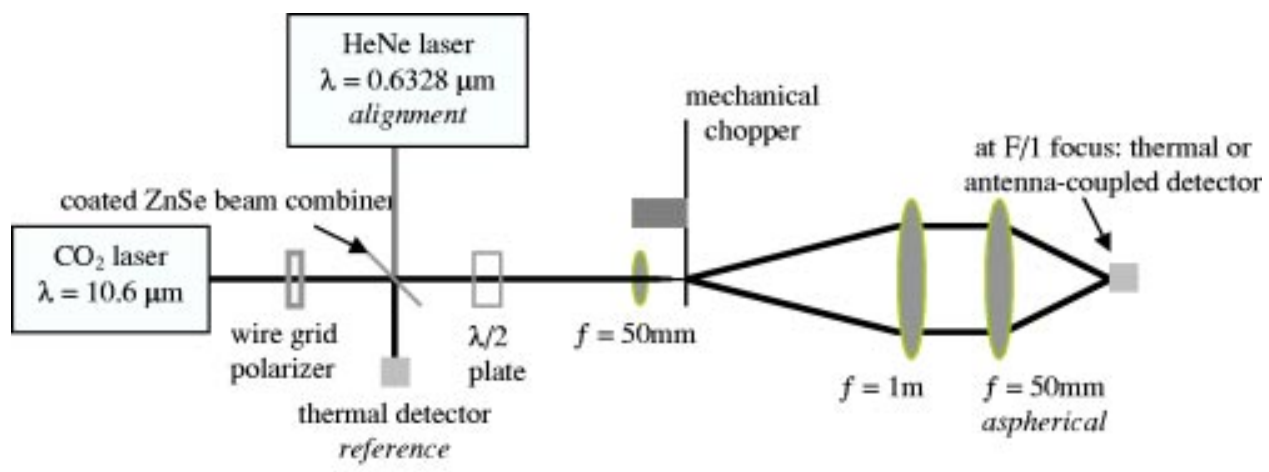

Fig. 1 Schematic of the experimental optical system.

ite optical system operates at $F / 1$. Our rationale for incorporating other optics in this system is explained in the following paragraphs.

Since the output of the carbon dioxide laser is polarized, a wire grid polarizer is used to control the laser power. A coated $\mathrm{ZnSe}$ beam combiner serves a dual purpose-it enables a visible helium-neon laser to exactly trace the $\mathrm{CO}_{2}$ beam, and it directs a portion of the IR radiation to a reference thermal detector. The visible laser is then used to transversely align the optical system via autoreflection from the lens surfaces. (All lenses are composed of zinc selenide; therefore, the optical system is transparent in both spectral regions.) The reference detector is essential in tracking the IR laser power fluctuations; the significance of this reference signal is covered in detail in Sec. 4.

Since the antenna-based detectors under test have a polarization response particular to their geometry, it is desirable to rotate the linear laser polarization with a zinc sulfate half-wave plate (a quarter-wave plate can be substituted for the half-wave plate if circular polarization is desired for the detector characterization). This optic is also transmissive in the visible and has little influence on the transverse alignment. A 50\% duty-cycle mechanical chopper is included in this system to modulate the laser power so that a lock-in amplifier can be used to collect the detector output signal.

Aside from its utility during the optical system alignment, the visible laser beam is also used to more easily locate the microscopic detectors under test at the $F / 1$ focus. Of course, there will be chromatic aberration separating the red $\mathrm{HeNe}$ focus from the IR $\mathrm{CO}_{2}$ focus. Thus, the visible and IR foci are separated by a fixed amount that can be reconciled during data acquisition-a detector can be located at the visible focus, and then defocused to obtain maximum response from the IR radiation alone.

However, for initial laser beam waist characterization, a knife edge is placed at the $F / 1$ focus. A thermal detector, identical to the reference thermal detector, is adhered to the knife edge, and high-resolution stepper motors (Melles Griot nanomovers) are used to transversely and axially scan the knife edge through the focal region. The maximum resolution of these stages is $1 \mu \mathrm{m}$ and their full range of motion is $25 \mathrm{~mm}$, more than sufficient to scan the entire focal region.

Data are taken to locate the exact laser beam waist by the following procedure. The knife edge transversely scans the focal region. Initial resolution is $100 \mu \mathrm{m}$ over a range of $2 \mathrm{~mm}$. The resolution is then decreased to $25 \mu \mathrm{m}$ over a range of $1 \mathrm{~mm}$. As the exact focal region becomes obvious, the next iteration takes data at 5- $\mu \mathrm{m}$ steps over a $500-\mu \mathrm{m}$ range, finally down to the lowest resolution of the stage, $1-\mu \mathrm{m}$ resolution over a $200-\mu \mathrm{m}$ range. These highresolution scans are extremely time-consuming, taking over $8 \mathrm{~h}$ to scan the focal volume, but they yield the most accurate data attainable with this system. Data from scans of the laser beam waist are compared in Sec. 5 .

\section{Multivariate Technique Applied}

We used a multivariate statistical analysis based on a principal component approach (PCA). The inputs to this algorithm are the knife-edge scan data. The input data set is noted as

$E=\left\{E\left(z_{1}\right), E\left(z_{2}\right), \ldots, E\left(z_{N}\right)\right\}$,

where $E\left(z_{i}\right)$ is the knife-edge scan data set, and $N$ is the number of scans taken. Data are taken transversely through the beam, at axial position $z_{i}$. Therefore, the data set consists of the laser power as a function of the transverse knife-edge position. The data are assumed to have a zero mean. Each of these scans consists of $M$ points and is affected by noise in the laser output. For each data set, a reference signal is simultaneously collected,

$R=\left\{R_{1}, \ldots, R_{N}\right\}$,

where $R_{i}$ consists of $M$ points, according to the range of knife-edge scan. The PCA treats each set of data as a multivariate random signal of $N$ dimensions. For the set of data collected, it is possible to form the covariance matrix for each data set. Principal components $Y_{\alpha}$ are linear combinations of original variables but there is no correlation among them (their covariance matrix is diagonal). The coefficients that form these linear combinations are given by the eigenvectors of the covariance. ${ }^{5}$ Moreover, the variances of principal components $\lambda_{\alpha}$ are arranged in decreasing order. These variances are the eigenvalues of the covariance matrix.

Original variables can be reconstructed for relevant principal components by means of 
$Y_{\alpha}=\sum_{i=1}^{n} e_{\alpha}(i) E\left(z_{i}\right) \Leftrightarrow E\left(z_{i}\right)=\sum_{\alpha=1}^{N} e_{\alpha}(i) Y_{\alpha}$,

where $e_{\alpha}(i)$ is component $i$ of the eigenvector $\alpha$ of the original data covariance matrix. ${ }^{5}$ Another important parameter of this decomposition is the value of $\Omega_{\alpha}$. It is defined by

$\Omega_{\alpha}=\frac{\lambda_{\alpha}}{\sum_{i=1}^{N} \lambda_{\alpha}}$

This parameter represents the portion of variance explained by principal component $\alpha$ in the total data set. Normally only a few of these terms represent most of the total variance data. This is the normal application of principal components-they decrease the degrees of freedom needed to describe a signal.

We applied this method in previous applications to the characterization of noise structures. We developed a method to automatically group different principal components into relevant spatial-temporal structures. The method is based on the study of the uncertainties in the variance of principal components. If the variance of two principal components overlaps, within uncertainty, they should been taken as a whole, because they explain the same amount of data variance. Any unitary linear combinations of these terms explain the same variance. In this case, both determine up rotations. ${ }^{6}$ They are correlated only in a "mean value" sense. The portion of data set reconstructed by these terms via Eq. (3) can be treated as a whole. The same reasoning may be applied to any continuos overlapping set of principal components. ${ }^{6}$

\section{Reference Signal}

The statistical analysis method described in the previous section is applied to a reference signal of the $\mathrm{CO}_{2}$ laser used in the detector characterization system. The sampling rate of the data is one second and the number of data points collected is 30,000 . A set of variables is constructed taking 100 subsets of 300 points. After applying the method of Sec. 3, the data are rearranged in their original format of 30,000 time series data for comparison. Figure 2 shows the varying reference signal. The extracted eigenvalues with their uncertainties are shown in Fig. 3. It is possible to recognize three different types of processes. The first process is comprised of the first two principal components, which includes $84.54 \%$ of the total data. The second process takes $14.39 \%$ of the total data and the third process uses $1.06 \%$ of the total data. After this classification, three different subsets are reconstructed from the original data, each consisting of the reference signal reconstructed only with relevant principal components. It is possible to construct the evolution of the "mean signal" by subtracting the summation of the three previous noise processes in the original data. The results are shown in Fig. 4.

The mean and standard deviation for each of these subsets are shown in Table 1. The mean and standard deviation of the "mean signal" fluctuation is also given. Process 1 is responsible for most of the reference signal fluctuation.

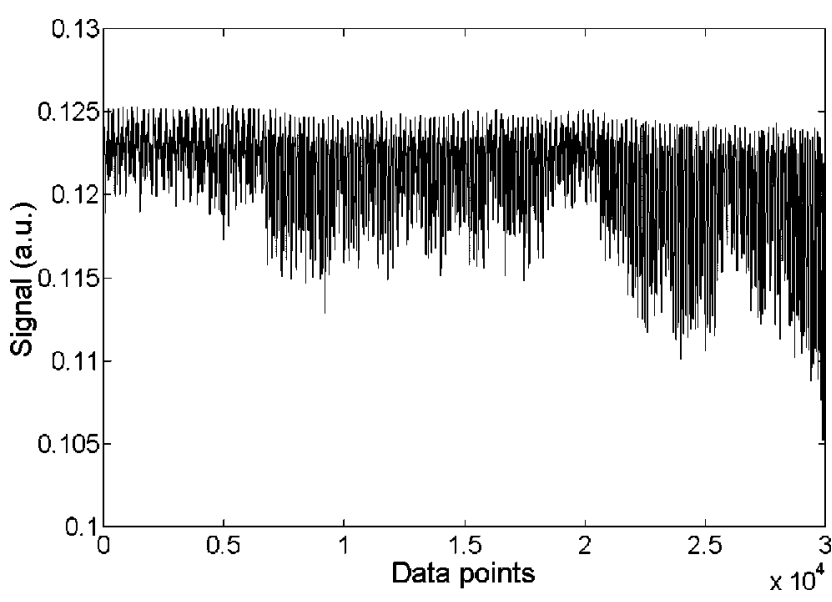

Fig. 2 Fluctuations of laser signal with time at the reference detector.

To obtain more information, the square root of the power spectrum density (PSD) is calculated for each noise processes. The results are shown in Fig. 5. A dependence on the temporal frequency is evident. This figure shows three main frequencies corresponding to a period of approximately 200,100 , and $50 \mathrm{~s}$, respectively. The most prevalent is the frequency associated with process 1 ; its period is $100 \mathrm{~s}$. Process 2 is associated with frequencies of period 200 and $50 \mathrm{~s}$. Process 3 resembles the behavior of a white noise process. These numbers suggest that the reference signal is affected by harmonics of a principal signal with a 100-s period, that is, around $1 \mathrm{~min}$ and $34 \mathrm{~s}$. This is precisely the cycling frequency of the cooler used to cool the $\mathrm{CO}_{2}$ laser. Therefore, thermal variation of the cooler is the main source of the laser power fluctuations. However, while frequencies are very well defined, the amplitude seems to be a random variable (see Fig. 4).

In addition, a more thorough characterization of process 3 and its mean value deviation are explored in Figs. 6 and

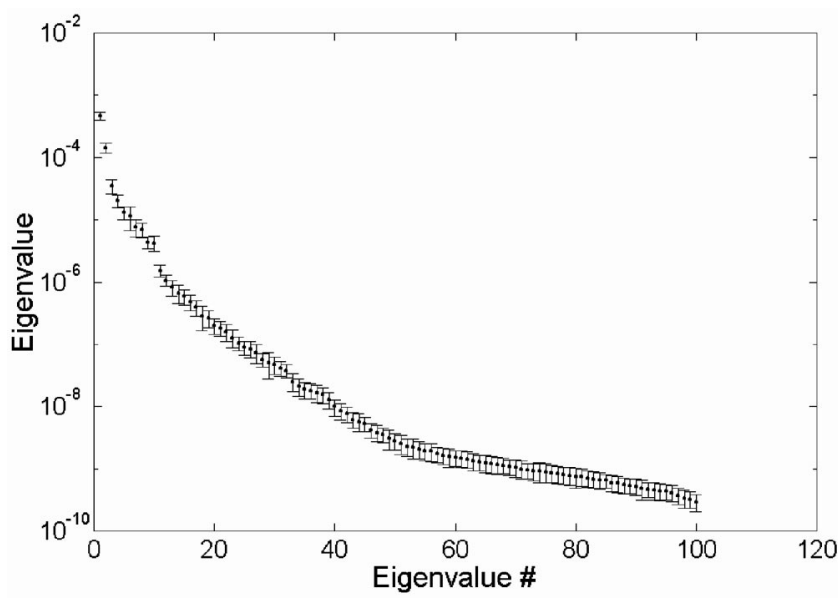

Fig. 3 Decomposition of the knife-edge scan data into its principal components. Three structures appear in the data set. The first consists of two isolated principal components, which represent the knife-edge itself. The second structure has mixed principal components, and a third has a large number of principal components that are associated with a single noise process. 

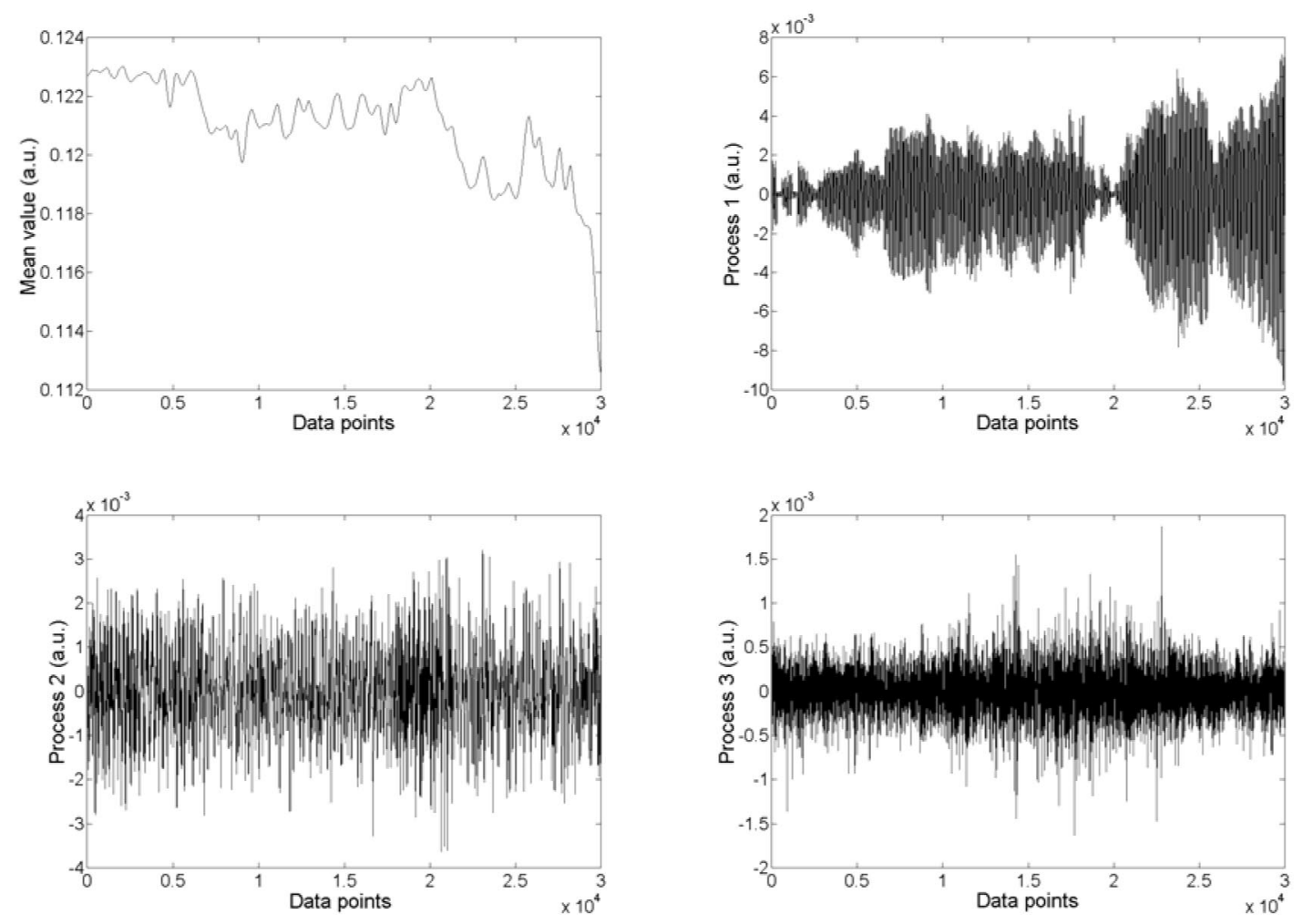

Fig. 4 Reconstruction of laser signal with the processes determined by PCA. Mean evolution of the laser signal (upper left) and first (upper right), second (lower left), and third noise processes (lower right).

7. Process 3 is could be possibly related to natural noise of the laser emission and detector noise. The histogram of this signal is plotted in Fig. 6. It is a very well defined probability distribution. The deviation of mean values has no particular feature, but looks like a random departure. With this in mind, we calculated the histogram of "jumps" between consecutive points in the mean value to test if they follow a reasonable probability distribution for a random departure. The results are also shown in Fig. 6. Since the goal of this paper is not to completely characterize the laser fluctuations, but to filter them from data set, no further studies of this process were conducted.

The laser signal appears to be characterized by a complex stochastic process with a mean value whose difference between consecutive points has a probability distribution. In addition, there are two processes with harmonic behavior. Finally, a white noise term is added that follows a welldefined probability distribution. In the next section, we apply these statistical techniques to the scan data sets to filter the fluctuations of laser, in an effort to reconstruct the location of the beam focus and beam shape with high fidelity.

Table 1 Values of mean and standard deviation.

\begin{tabular}{lcclll}
\hline \hline & Reference & $\begin{array}{c}\text { Mean } \\
\text { Values }\end{array}$ & $\begin{array}{c}\text { Process } \\
1\end{array}$ & $\begin{array}{c}\text { Process } \\
2\end{array}$ & $\begin{array}{c}\text { Process } \\
3\end{array}$ \\
\hline Mean & 0.1210 & 0.1209 & 0 & 0 & 0 \\
$\begin{array}{l}\text { Standard } \\
\text { deviation }\end{array}$ & 0.0030 & 0.0017 & 0.0024 & 0.0010 & 0.0003 \\
\hline \hline
\end{tabular}

\section{Subtraction of Noise from the Knife-Edge Scan Data Set}

After studying the stochastic fluctuations of the direct laser output, another data set is taken. It consists of the following simultaneous measurements.

1. 150 transverse knife-edge scans across the laser beam, taken with $2-\mu \mathrm{m}$ axial ( $z$ direction) steps; each scan consists of 200 points $(200-\mu \mathrm{m}$ transverse range) with a transverse step of $1 \mu \mathrm{m}$.

2. a reference signal collected directly from the laser output.

This reference signal is taken simultaneously with the knife-edge scans to normalize the scans. This normalization is taken as the ratio of the raw knife-edge scans (item 1) to the reference signal (item 2). This operation mitigates the mean value instability of laser signal fluctuations over the entire data set. The remaining fluctuations consist of noise with zero mean and high frequency, which are introduced by laser processes 1, 2, and 3. This noise will be filtered by the PCA procedure, since it is small variation of the knifeedge scan data.

The following filtering procedure is applied to the knifeedge scan data.

1. Each scan is differentiated to obtain a laser beam profile. This procedure enhances noise structures that may have been hidden in the noise of the data set. It could come from sources other than laser fluctua- 

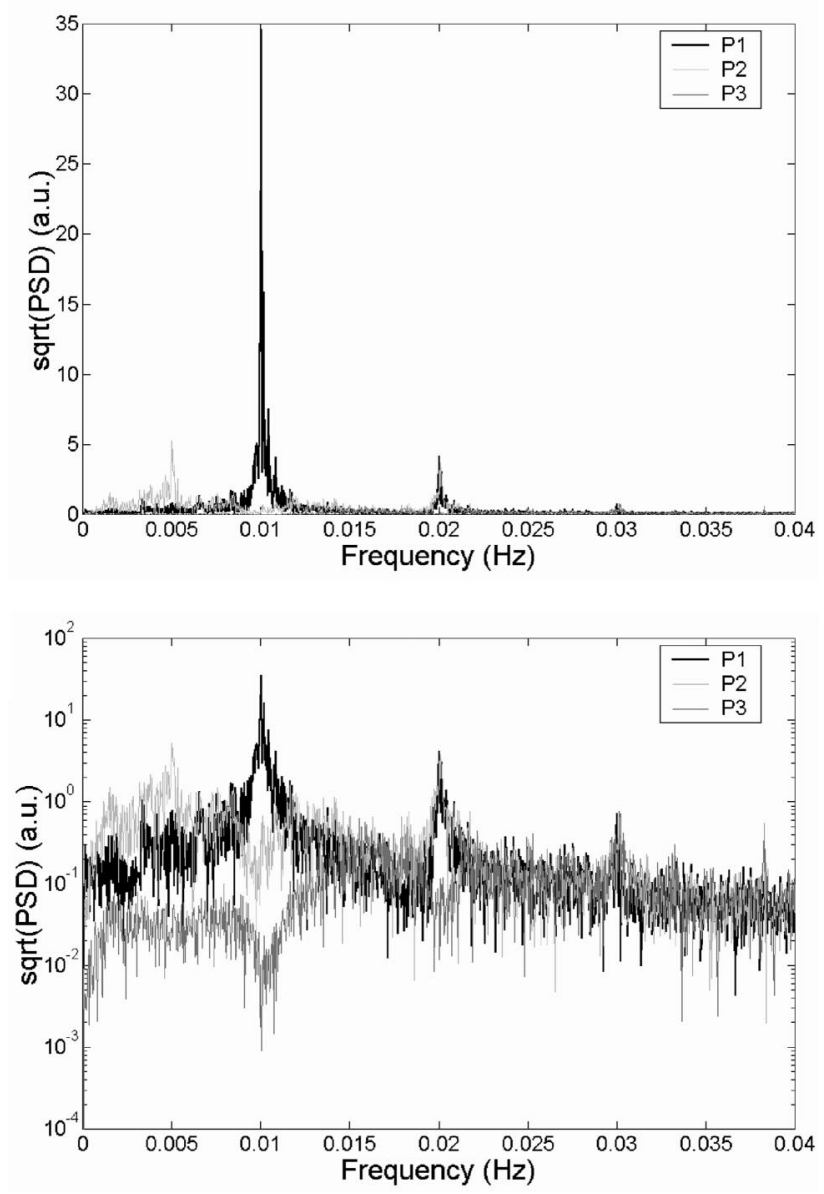

Fig. 5 Amplitude spectrum of noise processes 1 (P1), 2 (P2), and 3 (P3) in the reference signal. Linear scale (upper) and logarithmic scale (lower).

tions, such as uncertainty in knife-edge position due to stage motor error. This data set is hereby termed the differentiated data set.

2. The PCA is applied to the differentiated data set. The variables of this data set are the optical power at each $z$ value. The covariance matrix of these variables is formed and its eigenvalues and eigenvectors are calculated. The principal components are calculated as shown in Eq. (3). Using the PCA as before, we classify these principal components into separate processes. From the PCA filtering of the reference signal, as described in Sec. 4, we know that the noise produced by the laser fluctuation will also affect the differentiated data set. The structure of the differentiated data set then consists of a low-frequency signal along the $z$ direction, due to the real differentiated scan data (the knife-edge data itself) and a random signal due to laser fluctuations. The expected structure of principal components is formed by one group that reproduces the differentiated knife-edge scan data and another group of these components that forms a single process. The latter group represents the laser noise fluctuations. We tested this structure
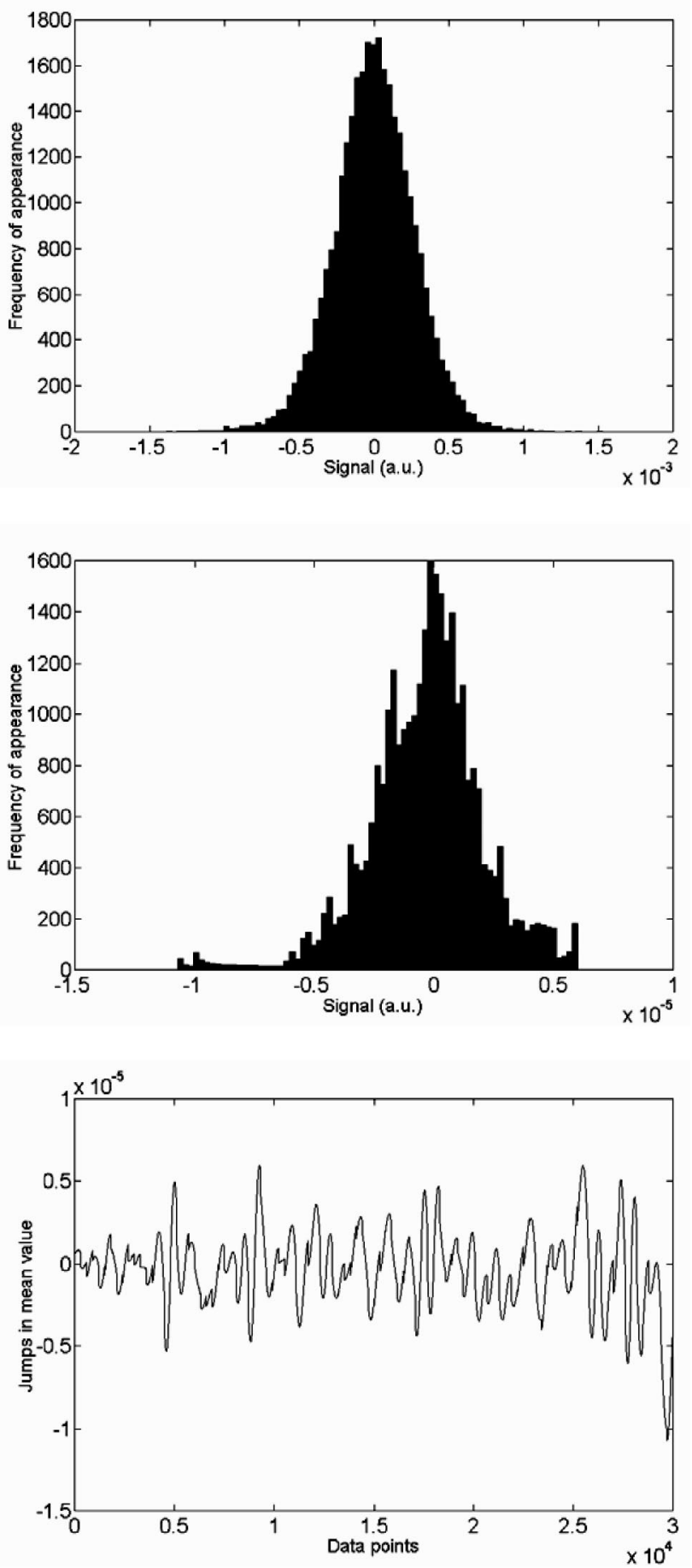

Fig. 6 Histogram of process 3 (upper). Also, the histogram (middle) and plot (lower) of the difference between consecutive points of the mean signal evolution.

with various data sets and it appears in all cases. Therefore, it is possible to automate this filtering routine in any data acquisition procedure.

3. The differentiated data set is then filtered the using the PCA rectification method, ${ }^{6}$ i.e., applying Eq. (3) by selecting a given subset of principal components. In this case, we reconstruct a new data set containing 

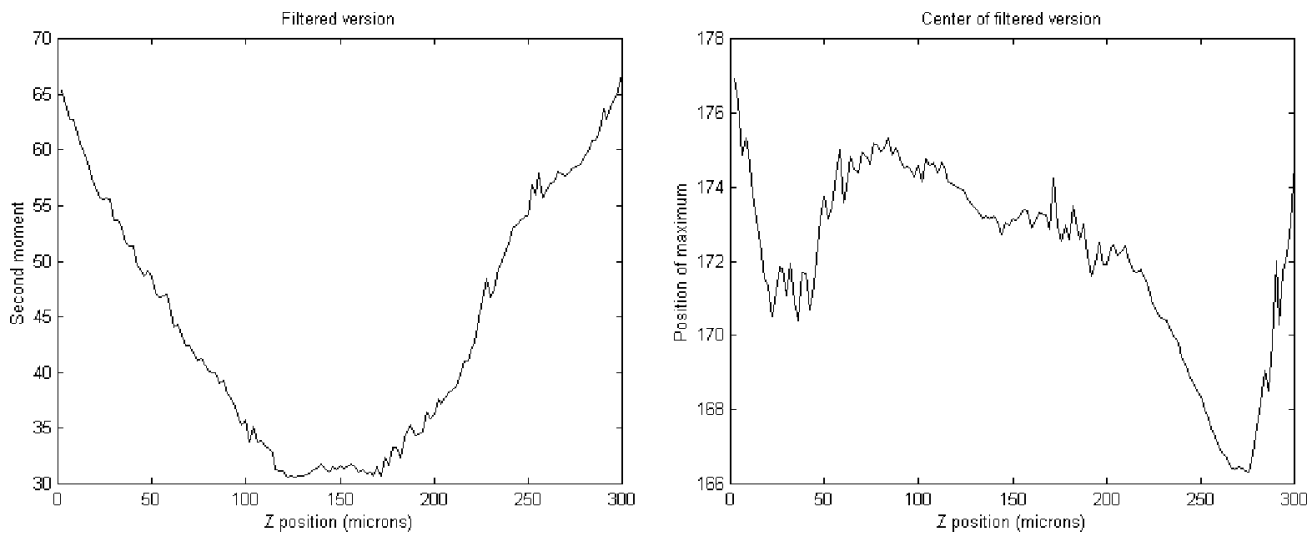

Fig. 7 Second (left) and first (right) moments of the laser beam after PCA filtering and after taking the ratio of the original data set to the reference signal. The moments are calculated as a function of the axial coordinate $z$.

only the relevant principal components associated with the original knife-edge data. These relevant principal components are those components that do not manifest the noise processes described step 2 of this filtering procedure. They are easily identified be- cause they explain most of the variance [Eq. (4)]. This new data set is called the filtered, differentiated data set.

4. Using the filtered, differentiated data set, the first-
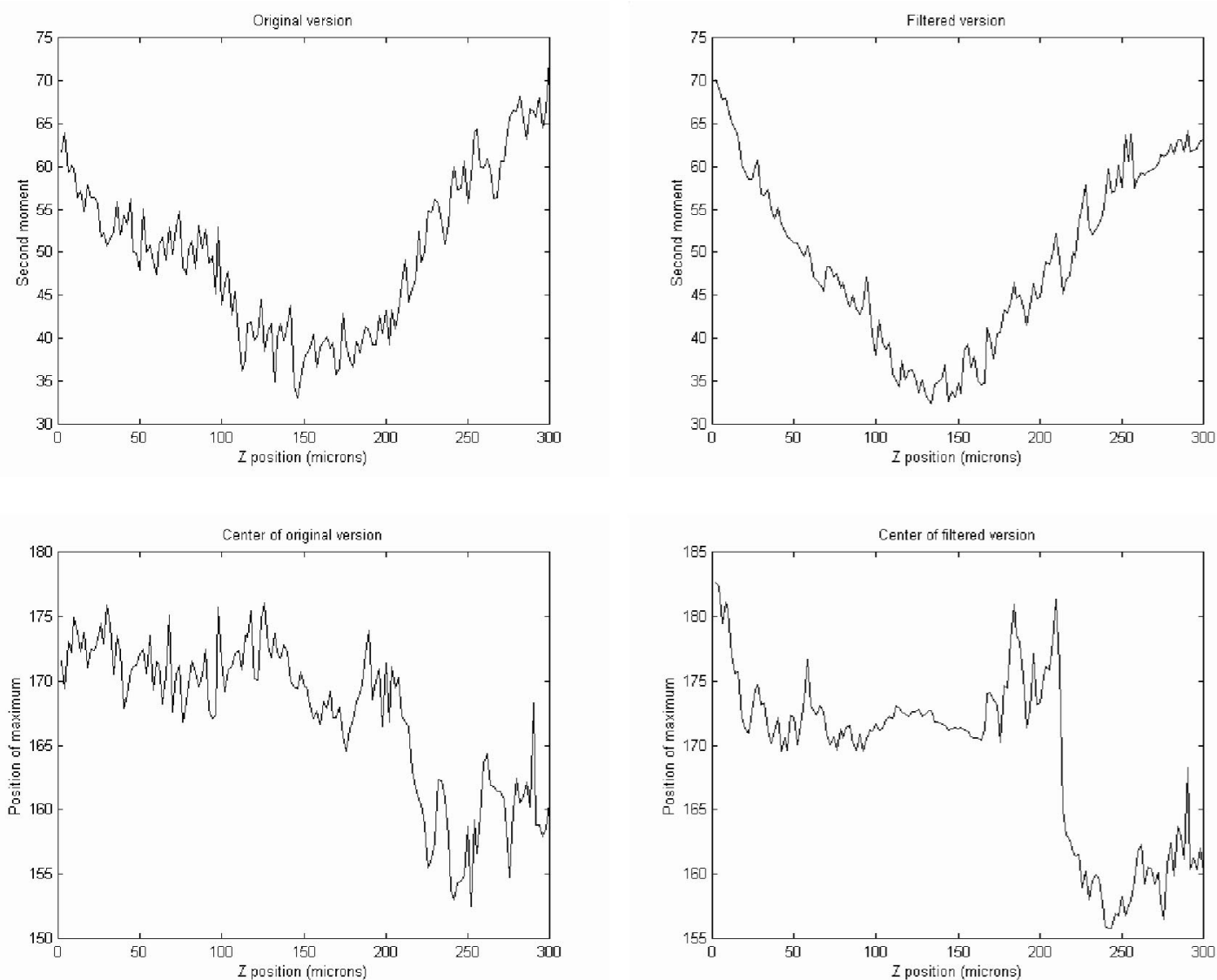

Fig. 8 Second (upper row) and first (lower row) moments of the laser beam. All data here are shown without PCA filtering. The left column shows data in which the ratio of the original data set to the reference signal has not been taken. The right column shows data in which this ratio has been taken. Only when the data are normalized to the reference signal and filtered via PCA is good accuracy obtained (compare these maps to Fig. 7). 

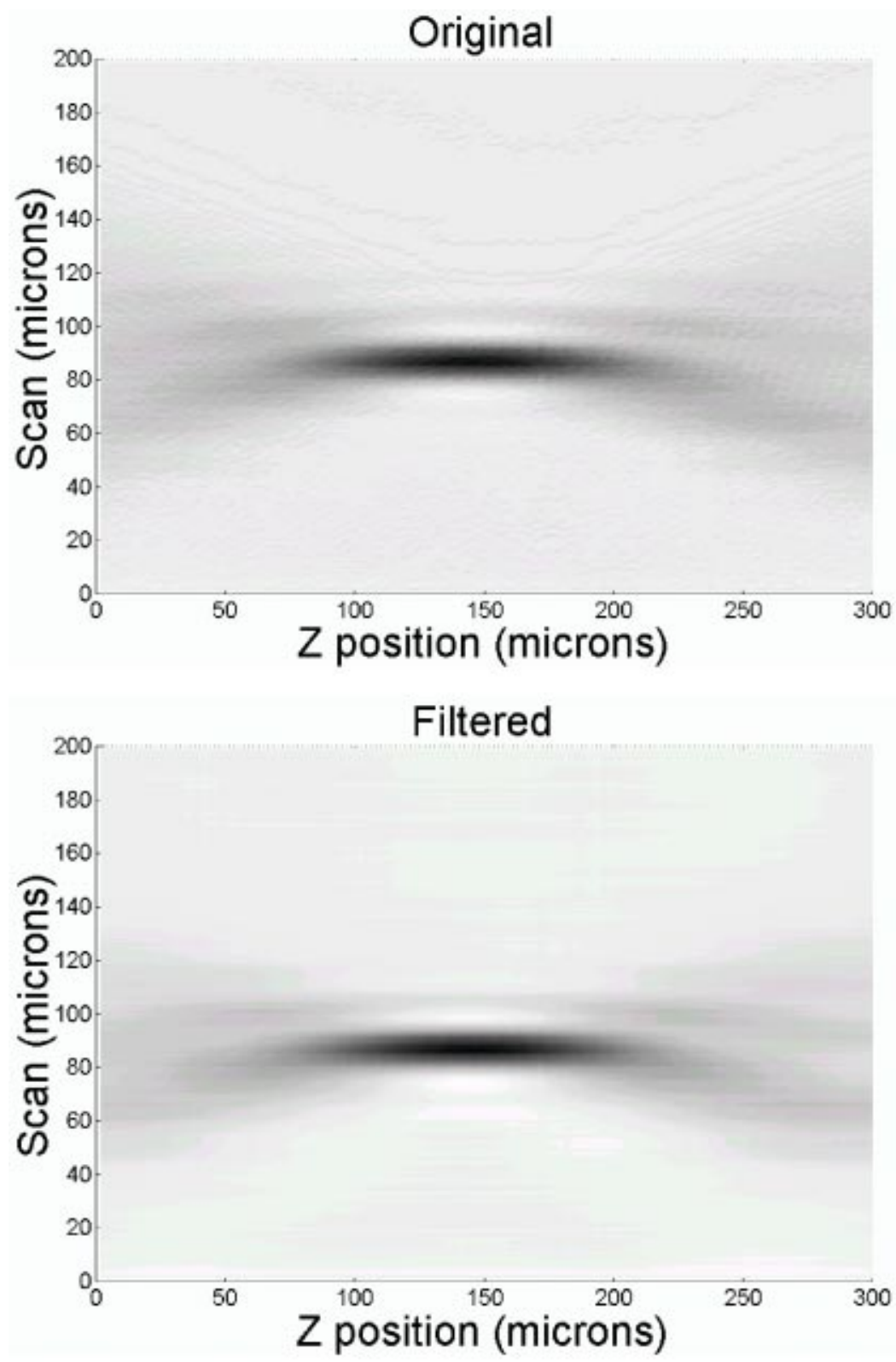

Fig. 9 Power distribution in the focal region with $1-\mu \mathrm{m}$ scan resolution across the beam and $2-\mu \mathrm{m}$ resolution along the laser beam axis. The lower portion of the figure shows filtered data; the upper portion shows unfiltered data.

and second-order moments of each knife-edge scan are calculated and plotted as a function of the $z$ (axial) coordinate.

5. The laser beam focus is calculated as the position of the minimum of the second-order moment. Due to the finite laser beam waist, this position is difficult to precisely locate. It is obtained by fitting the slopes of the second-order moment outside of beam waist to straight lines. Linear fitting produces two straight line equations: $\quad r_{\text {left }}=m_{\text {left }} z+b_{\text {left }}$ and $\quad r_{\text {right }}=m_{\text {right }} z$ $+b_{\text {right }}$. The intersection point of these lines, $r_{\text {left }}$ $=r_{\text {right }}$, locates the focus, $z_{\text {focus }}=\left(b_{\text {right }}-b_{\text {left }}\right) /\left(m_{\text {left }}\right.$ $\left.-m_{\text {right }}\right)$. It is possible to obtain an uncertainty for this focal position due to the uncertainty of the fit parameters to the straight lines. This uncertainty $u^{2}(z)$ is given by ${ }^{7}$

$$
\begin{aligned}
u^{2}(z)= & \left|\frac{1}{m_{\text {left }}-m_{\text {right }}}\right|^{2}\left[u^{2}\left(b_{\text {right }}\right)+u^{2}\left(b_{\text {left }}\right)\right] \\
& +\left|\frac{b_{\text {right }}-b_{\text {left }}}{\left(m_{\text {left }}-m_{\text {right }}\right)^{2}}\right|^{2}\left[u^{2}\left(m_{\text {right }}\right)+u^{2}\left(m_{\text {left }}\right)\right],
\end{aligned}
$$

where $u^{2}(x)$ represents the variance of variable $x$. 

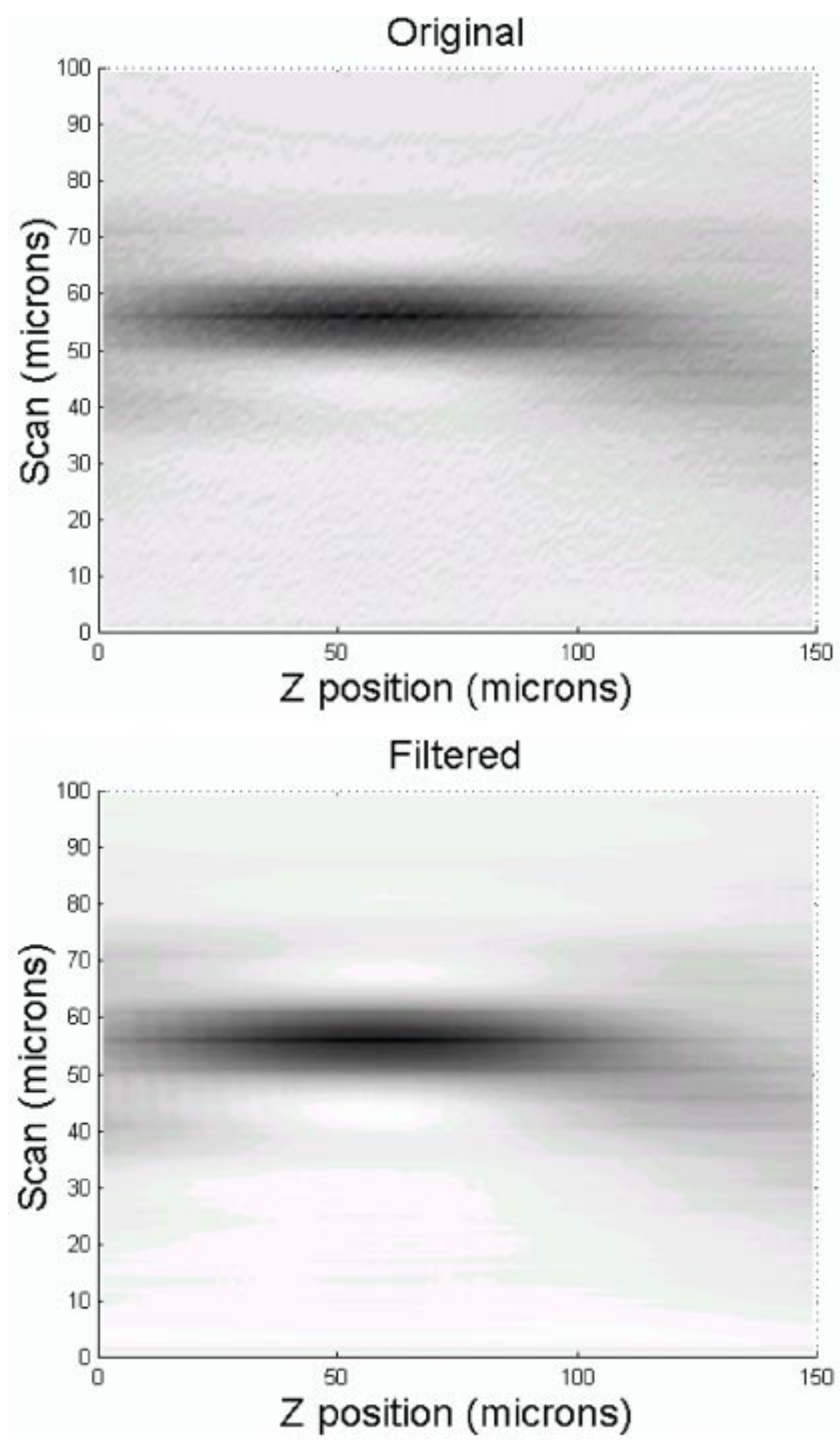

Fig. 10 Power distribution in the focal region with $1-\mu \mathrm{m}$ scan resolution across the beam and $1-\mu \mathrm{m}$ resolution along the laser beam axis. The lower portion of the figure shows filtered data; the upper portion shows unfiltered data.

This procedure is implemented by MATLAB software to automate the calculation of the focal position. Calculated second and first moments are shown in Fig. 7. Using these data, the best focus is calculated at $z=145 \pm 6 \mu \mathrm{m}$. For comparison, we make these calculations without applying this procedure, as shown in Fig. 8. The calculations are made without filtering via PCA, with and without taking the ratio of the knife-edge scans to reference signal (shown in the right and left columns of Fig. 8, respectively). Only by taking the scan to reference signal ratio and by filtering with PCA is it possible to locate the waist with accuracy. The laser irradiance in the focal region is shown in Fig. 9; the original, differentiated, but unfiltered scan data are shown in the upper map and the differentiated, filtered scan data are shown in the bottom map.

To enhance this noise for more complete filtering, a second data set with a resolution of $1 \mu \mathrm{m}$ is taken along the $z$ axis. The focal region is shown in Fig. 10. In these figures, the noise is even higher, composing the $2 \%$ of the total signal. The PCA is able to filter this noise and calculate 

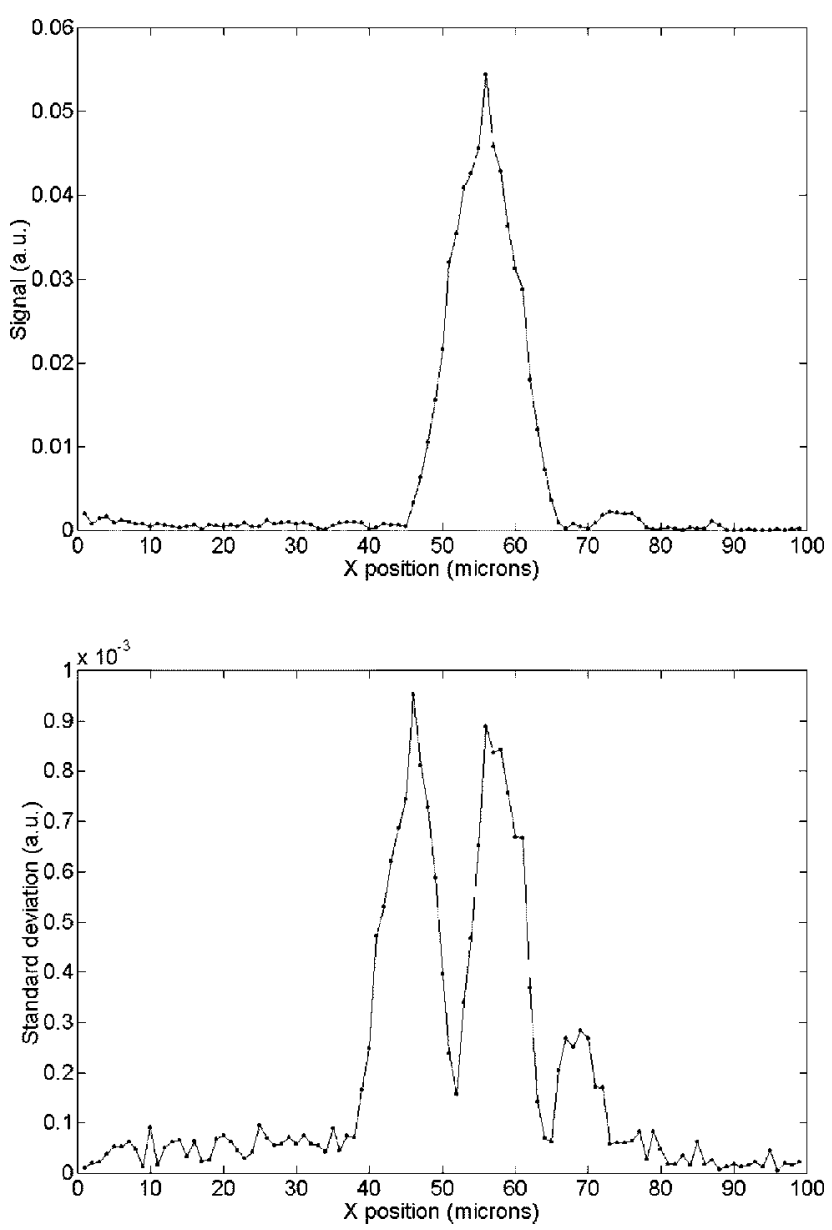

Fig. 11 View of the laser beam profile after filtering, at "best focus" position (upper) and the standard deviation (lower) for these data, taken with $1-\mu \mathrm{m}$ resolution for the transverse scan and along the beam axis. The optical system's diffraction-limited spot size of approximately $25 \mu \mathrm{m}$ is evident in the upper plot.

again the "best focus" position with accuracy (following step 5 of the data filtering procedure). In this case, the focus is located at an axial position of $65 \pm 4 \mu \mathrm{m}$. The beam shape is then calculated at this focus position using a filtered, differentiated data set. The uncertainty in the "best focus" position gives a region in which the focus is localized, rather than an unrealistic single point focus. Then, an uncertainty is calculated, taking into account only the measured data within this focal region. For each point of the scan, the uncertainty is calculated as the standard deviation of the beam signals at that point. These results are shown in Fig. 11. The standard deviation is lower at the maximum beam signal and high at both sides, illustrating the increase of the beam width at both sides of "best focus" position. It is possible to see that the beam size is at the diffraction limit of the optical system (approximately $25 \mu \mathrm{m}$ ), thereby characterizing and validating the accuracy of the alignment.

\section{Conclusions}

A method to align and calculate the "best focus" of a $\mathrm{CO}_{2}$ laser in an IR detector characterization system was developed. This procedure accounts for fluctuations in the laser signal. The technique used here is a multivariate statistical analysis based on a PCA. The method enables the laser signal to be modeled, and different types of noise can be identified. A majority of the noise comes from the laser cooling cycle. Changing cooler conditions is an expensive and impractical solution, because thermal fluctuations will always remain, although they may be mitigated by a more efficient cooler. Instead, the PCA is implemented to automatically filter this noise. This enables characterization of the optical system alignment and accurate calculation of the laser beam moments. Using these data, the laser beam waist location can be easily and accurately calculated. Finally, the beam shape can be plotted in the vicinity of the "best focus" position to determine information about the quality of the optical alignment. In our case, the laser beam spot size is at the diffraction limit of the optical system. The high degree of accuracy with this simple approach opens other possibilities, such as modeling the depth of focus, taking into account lens parameters of the optical system.

\section{Acknowledgments}

This work has been partially supported by project TIC2001-1259 of Minister of Science of Technology of Spain "Optical Antennas."

\section{References}

1. C. Fumeaux, G. Boreman, W. Herrmann, F. Kneubühl, and H. Rothuizen, "Spatial impulse response of lithographic infrared antennas," Appl. Opt. 38, 37-46 (1999).

2. J. Alda, C. Fumeaux, I. Codreanu, J. Schaefer, and G. Boreman, "A deconvolution method for two-dimensional spatial-response mapping of lithographic infrared antennas," Appl. Opt. 38, 3993-4000 (1999).

3. I. Codreanu and G. D. Boreman, "Integration of microbolometers with infrared microstrip antennas," Infrared Phys. Technol. 43, 335344 (2002)

4. F. J. Gonzalez, M. A. Gritz, C. Fumeaux, and G. D. Boreman, "Two dimensional array of antenna-coupled microbolometers," Int. J. Infrared Millim. Waves 23(5), 785-797 (2002).

5. D. F. Morrison, Multivariate Statistical Methods, 3rd ed., Chap. 8, McGraw-Hill, Singapore (1990).

6. J. M. López-Alonso, J. Alda, and E. Bernabeu, "Principal components characterization of noise for infrared images," Appl. Opt. 41, 320331 (2002).

7. ISO, Guide to the Expression of Uncertainty in Measurements, International Organization for Standarization, Geneva (1993).

José M. López-Alonso was with the Thermovision Laboratory of the Centro de Investigación y Desarrollo de la Armada from 1994 to 2002 developing figures of merit for the characterization of IR and visible images. He received his Lic degree in physics from the University Complutense of Madrid in 1994 and his PhD degree in 2002 on the topic of characterization of thermal cameras. He is with the Optics Department of the University Complutense of Madrid. He has been the Spanish delegate for the TG12 group of the North Atlantic Treaty Organization (NATO) devoted to the characterization of thermal imagers. In 2002 he was a member of the NATO ET024 group, dealing with broadband and multiband IR imaging systems. He has been also involved in the development of European EUCLID programs on the electro-optics topic.

Brian Monacelli: Biography and photograph not available.

Javier Alda has since 1985 been with the Optics Department, the University Complutense of Madrid, Spain, where he is a professor with the School of Optics. He received his PhD degree in 1988 in the field of laser beam characterization. He has been a visiting researcher at CREOL/School of Optics of the University of Central Florida for a more than 2 years. He has also been with the Ginzton Laboratory, Stanford University. His current research interests in- 
clude the characterization of noise and the performance of IR and visible cameras. He is now conducting a research project on optical antennas in the visible and near IR.

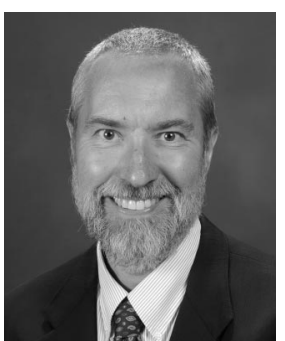

Glenn D. Boreman is Trustee Chair Professor of Optics, Electrical Engineering, and Physics at the College of Optics and Photonics (CREOL) at the University of Central Florida. He received a BS from the Institute of Optics, University of Rochester, and a PhD from the Optical Sciences Center, University of Arizona. He has been a visiting scholar at Imperial College in London, the Swiss Federal Institute of Technology (ETH) in Zürich, and the Defense Research Agency (FOI) in Linköping, Sweden. Dr. Boreman currently serves as the Editor-in-Chief of OSA's journal Applied Optics, and is a past member of the SPIE Board of Directors. He is coauthor of the graduate textbook Infrared Detectors and Systems, author of Modulation Transfer Function in Optical \& Electro-Optical Systems, and Basic Electro-Optics for Electrical Engineers. He has published more than 100 articles in the areas of infrared detector and focalplane analysis, optics of random media, infrared scene projection, and transfer-function techniques. Dr. Boreman is a Fellow of SPIE and OSA. He and two of his students received the 1995 Kingslake Medal from SPIE. 